\title{
Présentation de deux manomètres de précision pour le contrôle piézométrique des débits et la mesure de variations rapides de pression
}

\author{
Two precision manometers \\ for piezometric discharge control and the measurement \\ of rapid pressure variations \\ PAR P. BOURGUIGNON \\ INGENIEUR DES ARTS E'T MANUFACTURES \\ Chef de la Drvision « Essais Extérieurs 》 Du Service des Euvdes et Recherches \\ D'ERECTRICITÉ DE FranCE \\ English synopsis p. 636
}

\begin{abstract}
Au cours des essais qu'elle a effectués dans de nombreuses installalions hydroélectriques, la Division des Essais Extérieurs du Service des Etudes et Recherches Hydrauliques de lElectricité de France s'est attachée à améliorer certains procédés de mesure; elle a dú mettre au point ou perfectionner quelques appareils dont deux manomètres que nous nous permettons de présenter au Comité Technique de la Société Hydrotechnique de France.

Le premier appareil est utilisé pour la mesure de faibles différences de pression. Le deuxième, étudié avant la mise en service industrielle des appareils électroniques, était destiné à la mesure des pressions rapidement variables.

La caractéristique principale de ces manomètres devait être leur simplicité, allice à une bonne exactitude, obtenue au moyen de chaines métrologiques aussi courtes que possible.
\end{abstract}

\section{I. - MESURES DE FAIBLES DIFFÉRENCES DE PRESSION}

La détermination exacte de faibles différences de pression est souvent nécessaire; elle permet la détermination précise de la perte de charge en section courante d'un tronçon en alignement droit d'une conduite forcée, en vue de l'évaluntion d'un coefficient de rugosité, sans nécessiter l'emploi de longues canalisations de raccordement.

Elle permet surtout d'utiliser des dispositits piézométriques pour contrôler les débits dans les conduites industrielles. Bien que généralement exemptes de singularités importantes, ces dernières comportent néanmoins des convergents de faible contraction ou des coudes peu accusés qui engendrent, entre deux prises voisines, des différences de pression atteignant pour le débit maximum 30 à $50 \mathrm{~cm}$ d'eau. Les dénivellations correspondant aux débits fractionnaires de ces contrôles piézométriques nécessitent donc une précision de lecture de l'ordre de 1 à $2 \mathrm{~mm}$ d'eau, qui ne peut guère être obtenue avec le manomètre différentiel classique à colonnes de mercure.

Parmi les appareils les plus simples auxquels on pouvait songer pour satisfaire ces besoins, le manomètre différentiel à colonnes « air-eau » 
paraissait a priori posséder une sensibilité suffisante (1). Toutefois, son utilisation à la mesure de pressions fluctuantes, lui faisait perdre beaucoup de sa précision de principe, du fait de la compressibilité de l'air. En effet, si l'on soumet à des variations de pression plus ou moins rapides les deux enceintes auxquelles est raccordé un manomètre différentiel à colonnes « air-eau », constitué d'un simple tube en $U$ T renversé, on peut observer que les deux ménisques de séparation de l'air et de l'eau sont animés d'oscillations importantes, qui peuvent parfois ne présenter à première vue que peu de caractères communs. De ce fait, l'évaluation de la différence entre la position moyenne des deux ménisques peut être très imprécise. Au contraire, dans un manomètre différentiel à fluides incompressibles de même dimensions, soumis à des fluctuations de pressions analogues, les oscillations, d'ailleurs plus faibles, des ménisques s'effectuent en sens opposé mais restent proportionnelles entre clles: les lectures effectuées sur l'un des tubes peuvent être déduites, et contrôlées, par celles obtenues sur le second.

Il nous a done paru intéressant d'étudier les lois de réponse du manomètre différentiel à colonnes « air-eau », en vue de le doter d'un amortissement efficace et parfaitement linéaire des fluctuations les plus gênantes (2), qui se manifestent simultanément, et avec une égale intonsité, aux deux prises de pression auxquelles est raccordé le manomètre.

L'étude des lois de réponse du manomètre différentiel à colonnes « air-eau », aux fluctuations simultanées de la pression absolue, nous a conduits à un dispositif qui s'est révélć ésalement efficace à l'amortissement des fluctuations des différences de pression.

\section{Facteurs d'amortissement ides fluctuations DE PRESSION.}

Le théorème des forces vives appliqué aux masses fluides en mouvement dans un manomètre à deux fluides, comprenant l'appareil luimême et ses canalisations de raccordement aux prises de pression, permet d'établir l'équation différentielle du déplacement $x$ du ménisque de

(1) Nous avons écarté les manometres différentiels à denx liquides dont l'emploi dans les usines hydroélectriques est souvent délicat du fait de la turbidite des eaux et des difficultés de mise en station sous des pressions absolues souvent élevées.

(2) Nous avons été amenés à supposer, pour notre étude, qu'une fluctuation de pression pouvait être considérée comme la superposition de deux fluctuations distinctes; l'une qui se manifeste également sur les deux prises de pression du manomètre, l'autre qui n'affecte que la différence des pressions entre les deux prises. La première n'a en fait d'influcnce que sur les manomètres utilisant un fluide compressible. séparation des deux fluides sous l'influence des fluctuations périodiques de pression.

Cette équation différentielle, évidemment analogue à celle des mouvements pendulaires ou des circuits électriques, peut être mise sous la forme :

$$
\mathrm{L} \frac{d^{\prime} x}{d t^{2}}+\mathrm{R} \frac{d x}{d t}+\frac{1}{\mathrm{C}} x=h(t)
$$

Si l'on soumet le manomètre à une fluctuation de pression de pulsation w et d'amplitude $h$, l'amplitude $x$ (1) du déplacement du ménisque sera donnée par la relation (2)

$$
x=\frac{h}{\sqrt{\left(\mathrm{L} \omega-\frac{1}{C \omega}\right)^{2}+\mathrm{R}^{2}}}=\frac{h}{Z \omega}
$$

en désignant par $Z$ l'impédance du circuit équivalent. Le coefficient d'amortissement des fluctuations du manomètre sera défini par le produit $\mathcal{Z}$ (i).

I'expression analytique des diverses constantes intervenant dans les équations précédentes, en fonction des caractéristiques hydrauliques des manomètres usuels, est reproduite dans la figure $\mathrm{n}^{\circ} 1$.

Le terme de « self induction » $\mathrm{L}$, caractérise l'énergie cinétique des masses en mouvement; les frottements des fluides sur les parois sont représentés par le terme $R$, tandis que $\frac{1}{C}$ est relatif aux forces élastiques de rappel (3).

Parmi les trois moyens mis à notre disposition

(1) Dans notre expose, nous ne tiendrons pas compte du déphasage entre la fluctuation de pression et celle du ménisque. La tangente de cet angle serait évidemment donnée par la relation classique:

$$
\operatorname{tg} \varphi=\frac{R \omega}{L \omega^{2}-\frac{1}{C}}=\frac{R}{L \omega-\frac{1}{C \omega}}
$$

(2) Cette relation est celle qui relie la différence de potentiel $\mathrm{E}$ de pulsation $\omega$ appliquée aux bornes d'un circuit électrique d'impédance $Z$ à la quantité d'électricjté $q$.

(3) Ces expressions sont établies dans l'hypothèse d'un écoulement laminaire et permanent. Dans sa Contribution a l'Etude de la Mesure des pressions variables (Editions Hermann), M. Valembors a précisé les corrections qu'il y a lieu d'apporter aux constantes $I$ et $\mathbf{R}$ dans le cas d'un fluide incompressible en régime laminaire soumis à une variation de pression sinusoïdale.

Notre étude s'appuyant seulement sur l'ordre de grandeur de ces constantes, il ne nous a pas paru nécessaire de tenir compte de ces corrections, qui auraient d'ailleurs pour effet de majorer les valeurs que nous avons données. Leur omission est donc dans le sens de la sécurité. 


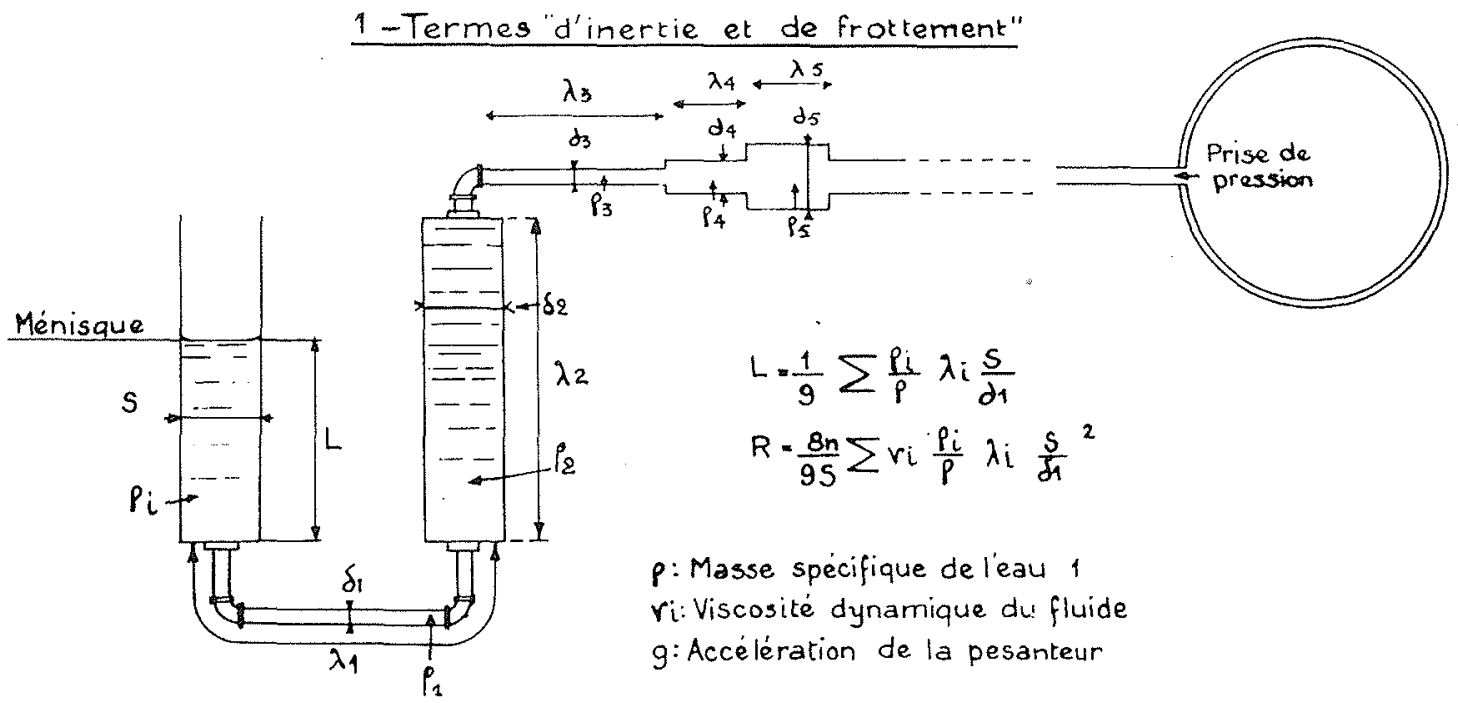

a-Tube piézométrique à deux fluides $\frac{2 \text {-Terme d'élasticité }}{b \text {-Manomètre differentiel à } 2 \text { fluides }}$
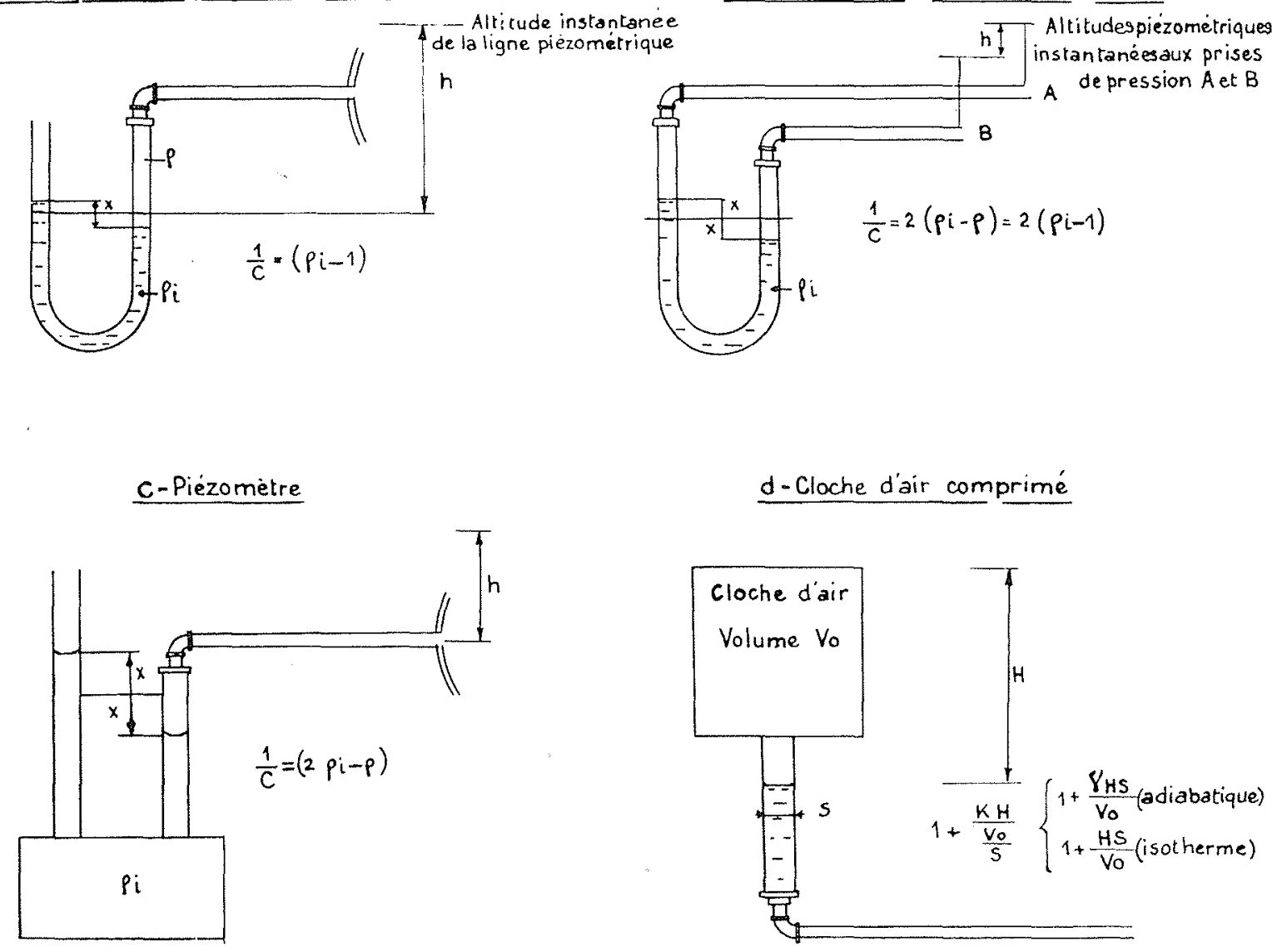

d-Cloche d'air comprimé

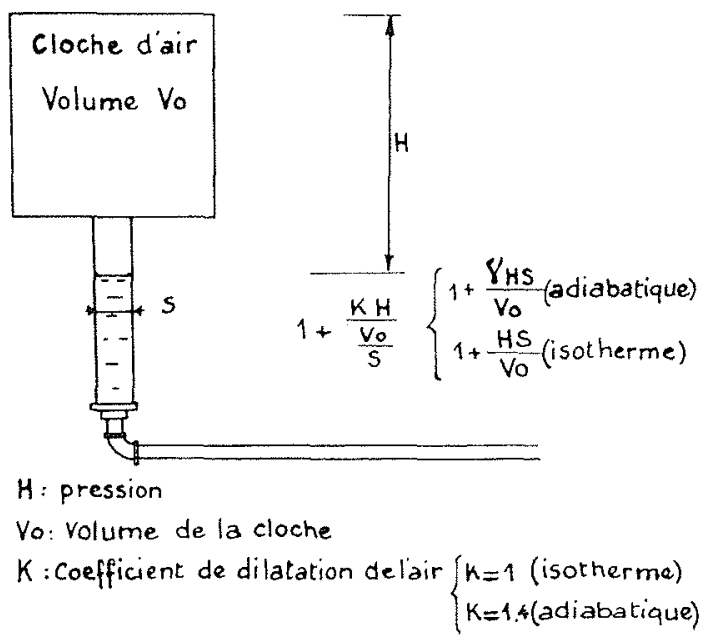

Fig. 1. - Constantes de ríponse d'un manomètre. 
pour réduire l'amplitude des oscillations simultanées des ménisques, nous avons éliminé a priori les frottements dans les canalisations.

Leur utilisation conduit en effet à des canalisations de faible section, susceptibles d'obstruction fréquente dans les installations industrielles aux eaux plus ou moins turbides.

En outre, une fuite même très légère, dans les moyenne d'une pression fluctuante que si, quelque soit le sens de parcours, les forces de frottement varient linéairement avec la vitesse, condition parfois difficile à réaliser.

L'utilisation de l'inertie de l'eau n'entraîne aucune erreur de principe sur la position moyenne du ménisque. Par contre, elle conduit rapidement à des dimensions excessives et corol-

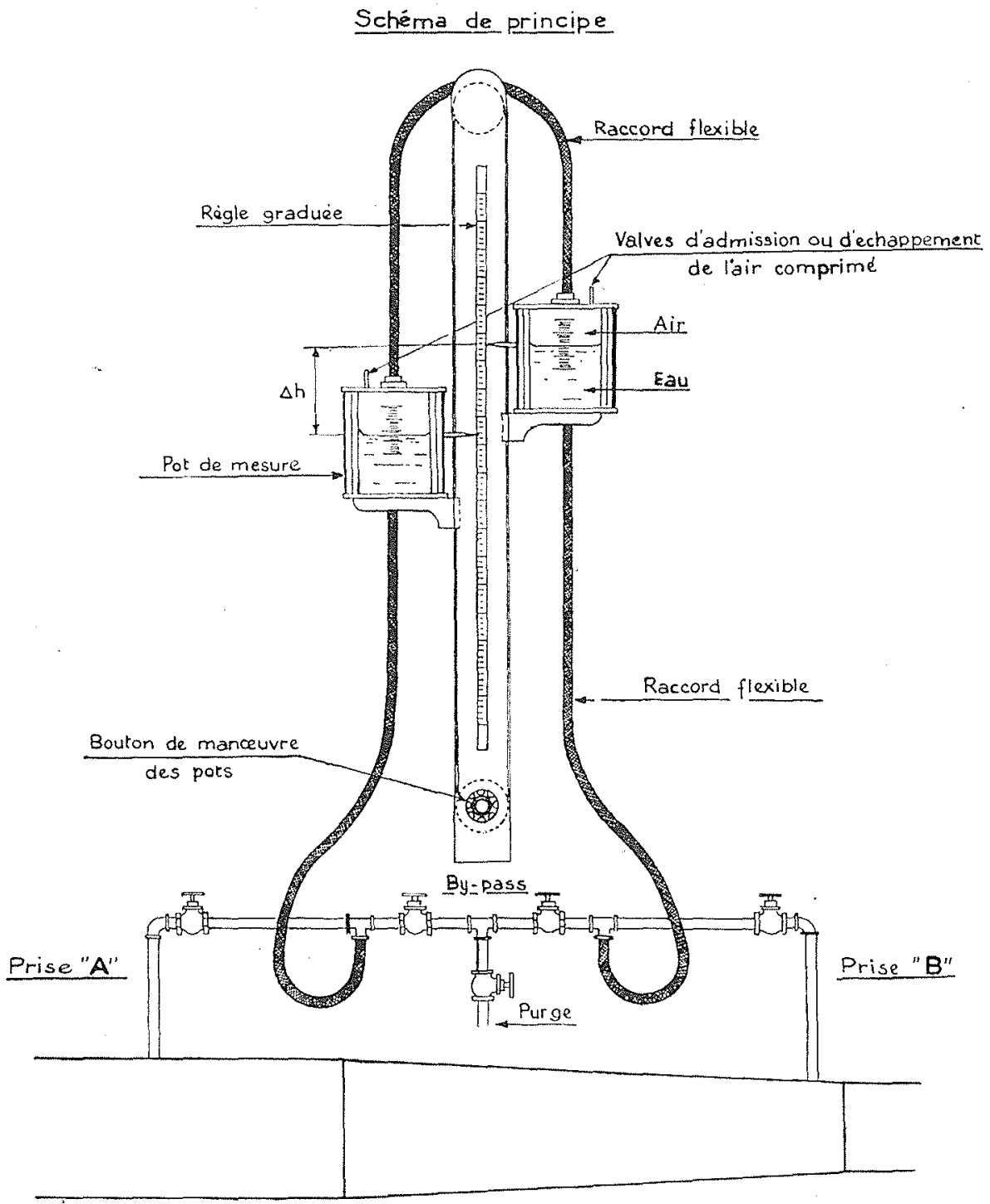

Fig. 2. - MaNoMEtRe DIFFÉRENThel a COLONNES AIR-EAU.

canalisations de raccordement d'un manomètre fortement amorti par frottement visqueux, peut entraîner des erreurs non négligeables (un débit de $1 \mathrm{~mm}^{3}$ par seconde, représentant environ 1 goutte par minute peut créer une erreur de l'ordre de $2 / 10 \mathrm{~mm}$ dans un manomètre comportant un dispositif d'amortissement par frottement dont la valeur de $R$ est 200 ).

Enfin, il y a lieu de rappeler qu'un manomètre ne donne d'une manière correcte la valeur lairement ì des temps prohibitifs de mise en équilibre de l'appareil.

C'est pour ces raisons que dans le manomètre différentiel à colonnes air-eau, utilisé par notre Service (le schéma de principe est reproduit dans la figure $n^{\circ} 2$ ), l'amortissement des oscillations des ménisques, dues aux fluctuations simultanées de la pression absolue, a été obtenue surtout par l'utilisation des forces élastiques de l'air comprimé. 
20 CaRACTÉRISTIQUES PRINCIPALES DU MANOMỲTRE DIFFÉRENTIEL A COLONNES 《 AIP-EAU 》.

\section{a) Amortissement des oscillations des ménisques}

Ainsi qu'on pent le voir sur la figure $\mathrm{n}^{\circ} 2$, les deux pots, dans lesquels évoluent les méniscrues, peuvent se déplacer également et en sens oppose, au moyen d'un dispositif à crémaillère. L'équilibre est réalisé lorsque par déplacement des pots, les ménisques viennent occuper en principe la mème position dans chacun d'eux. De cette manière, le volume d'air comprimé dans te manometre est trés réduit; compte tenu du volume compris dans la canalisation du raccordement supérieur, il représente une hauteur de 1 a 2 cn dans chacun des pots de mesure. Une fluctuation de pression de l'ordre de $10 \%$ de la pression absolue appliquée aux deux prises crée donc au maximum, quelle que soit la période, une amplitude de déplacement des ménisques inférieure à 1 ou $2 \mathrm{~mm}$ (1) ce qui, pour une pression absolue de $100 \mathrm{~m}$, représente un coefficient d'amortissement de 10.000 environ.

Pour les fluctuations des différences de pression appliquées aux manomètres, les forces élastiques de rappel sont réduites à la hauteur d'eau représentant la différence d'altitude des deux ménisques. La constante $\frac{1}{C}$ devient très faible et l'amortissement doit être assuré par les forces d'inertie et de frottement.

Compte tenu des dimensions de l'appareil, supposé réuni aux prises de pression par des canalisations de $10 \mathrm{~mm}$ de diametre et de $5 \mathrm{~m}$ de longueur, les constantes de l'écquation différentielle de réponse du manomètre sont:

$$
\begin{aligned}
& \mathrm{L}=60 \\
& \mathrm{R}=18 \\
& \mathrm{I}=2
\end{aligned}
$$

Une fluctuation de différence de pression de 5 cm d'amplitude et de $2 \mathrm{~s}$ de période entraîne donc une oscillation des deux ménisques, en sens opposé, et dont l'amplitude est voisine de

(1) Tn principe, il existe une pulsation critique, réalisant les conditions de résonance $\left(\mathrm{CL} \omega^{2}=1\right)$, pour laquelle l'amortissement est réduit à celui dû aux forces de frottement. La plage correspondant à l'abaissement sensible du coefficient d'amortissement est très réduite en principe (clle couvre un intervalle de période compris pratiquement entre 0,3 et $0,5 \mathrm{~s}$ ); jusqu'à présent ce phénomène de résonance n'a jamais été rencontré. Il serait d'ailleurs facile de l'éliminer pratiquement en faisant variex légèrement le volume d'air compris dans le manomètre, ce qui aurait pour effet de modifter la constante $1 / \mathrm{C}$ due à l'élasticité de l'air.
$1 / 10 \mathrm{~mm}$. Cette amplitude serait de $2 \mathrm{~mm}$ envron si la période des fluctuations de différence de pression était de $10 \mathrm{~s}$.

\section{b) Temps de mise en équilibre}

Les ménisques étant sans cesse ramenés à leur position initiale dans chacun des pots de mesure, grâce au déplacement de ces derniers, le volume d'eau déplacé dans les canalisations de raccordement au cours d'une mise en service est praticuement nul. Par suite, le temps de mise en équilibre est très réduit; en une vingtaine de secondes, les pots peuvent être amenés à moins de $1 \mathrm{~cm}$ près de leur position d'équilibre. Le réglage définitif s'effectue en moins d'une minute.

\section{$3^{\circ}$ Lectures.}

Pour les pressions inférieures à $20 \mathrm{~kg}: \mathrm{cm}^{2}$, les pots du manomètre en matière transparente portent une graduation sur la surface antérieure; les lectures sont effectuées par visée du plan inférieur du ménisque, ainsi que cela est représenté sur la figure $n^{\circ} 3$; grâce au coefficient d'amortissement élevé, ce ménisque est suttisamment stable pour que toute erreur de parallaxe soit éliminée.
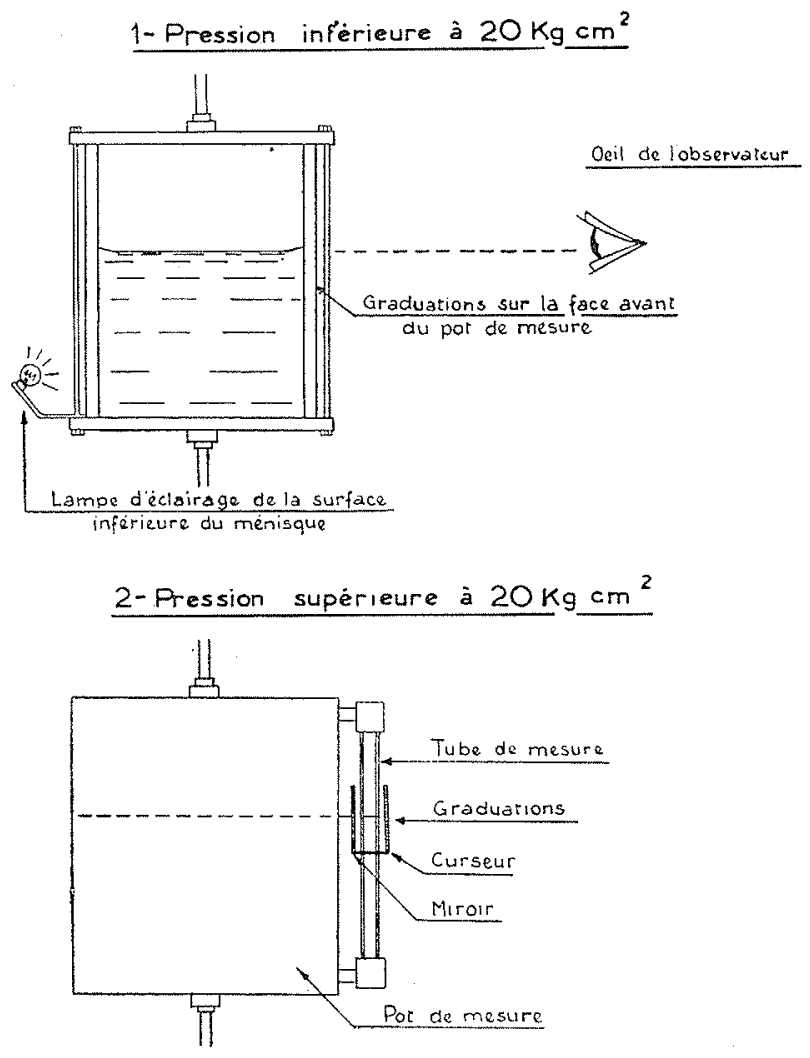

Fig. 3. - Lecture aU MaNomètre. 
Pour les pressions supérieures, chaque pot de mesure métallique est relié à un tube gradué sur lequel coulisse un curseur muni d'une lame de plexiglass appliquée contre la graduation du tube et comportant un trait horizonlal; un miroir parallèle à cetle lame, fixé au curseur et se déplaçant derrière le tube, permet d'éliminer l'erreur de parallaxe.

La position de chaque pot est repérée à $\pm 1 / 10 \mathrm{~mm}$ près au moyen d'un vernier.

4. MISE EN STATION.

\section{Compression de l'air}

L’air est comprimé à la partie supérieure du manomitre au moyen d'un compresseur hydraulique, analogue à celui utilisé dans la chambre d'air des installations de pompage et dont le schéma de principe est rappelé dans la figure $\mathrm{n}^{\circ} 4$.

\section{Purge et vérification du zéro du manomètre}

Les canalisations flexibles de liaison du pot au bloc robinet peuvent être réunies en parallèle à un circuit de purge d'air représenté dans la fisure $\mathrm{n}^{\circ} 5$.

L'eau de purge est admise par l'une ou l'autre des prises de pression el parcourt dans les deux sens les deux tuyaux flexibles, de manière à purger convenablement les troncons en contrepente, réalisés en vue de réduire l'encombrement de l'appareil.

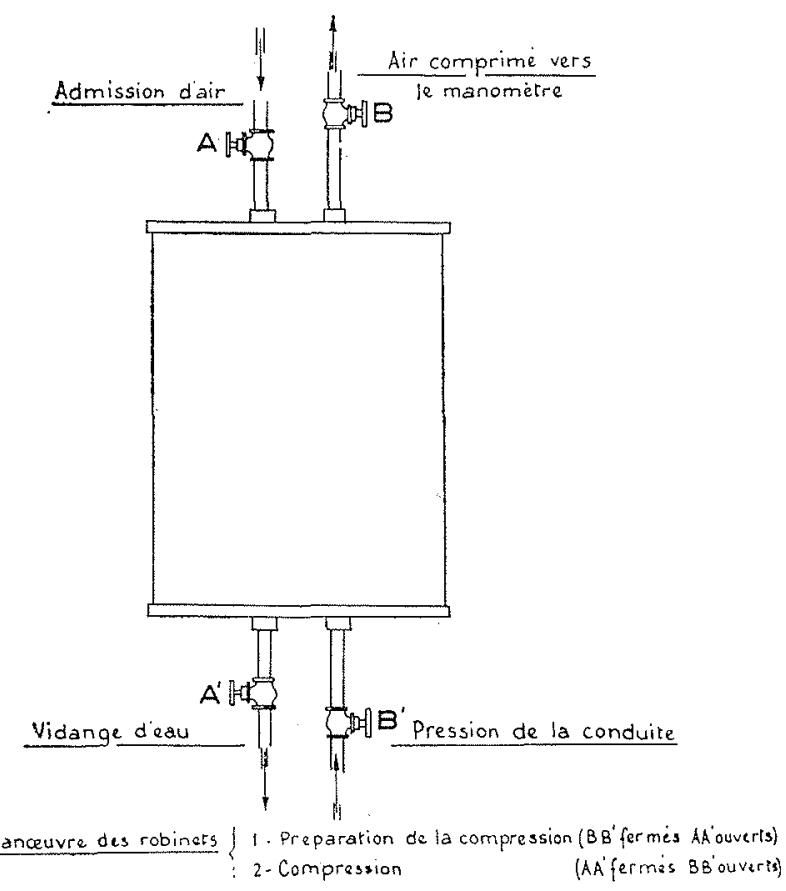

Fig. 4. - Compresseur d'air.

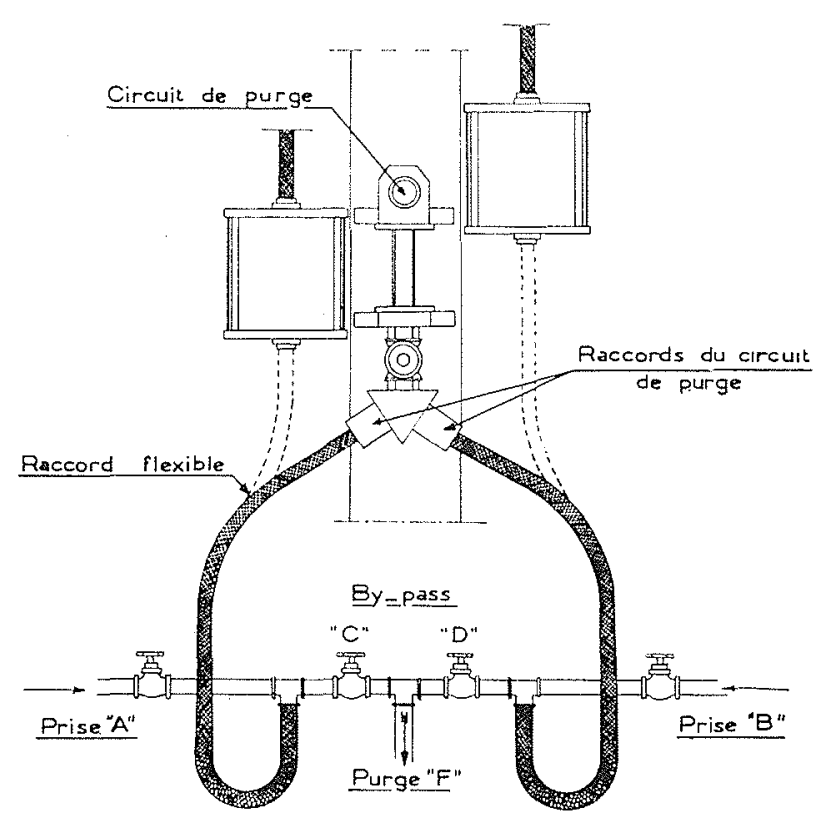

Fig. 5. - Punge D'ain d'Un MaNomètre.

L'efficacité de la purge du manomètre est contrôlée après le raccordement des canalisations aux pots de mesure; on peut vérifier en effet, que les ménisques se trouvent bien sur un même plan horizontal lorsque le by-pass des canalisations est ouvert.

\section{Exécution des relevés}

Pour faciliter la mise en service du manomètre, l'un des robinets d'isolement possède un étranglement susceptible de réduire la vitesse du déplacement des ménisques dans les pots au cours du dégrossissage de la mise en équilibre du manomètre. Lorsque les pots sont amenés au voisinage de la position correspondant à la différence de pression régnant entre les colonnes du manomètre, l'ouverture complète du robinet élimine l'étranglement.

\section{$5^{\circ}$ PrÉCISION DU MANOMÈTRE.}

Grâce à la stabilité excellente des ménisques et aux dispositions prises pour limiter les erreurs de parallaxe, la position des ménisques, par rapport aux pots mobiles, peut être repérée à $+1 / 10 \mathrm{~mm}$ près. Il en est de même de la position des pots par rapport au mat central. Toutes précautions ont été prises pour assurer le parallélisme parfait du déplacement des pots, ainsi que la verticalité du mat central, contrôlée par un fil à plomb de $1 \mathrm{~m}$ de hauteur.

Compte tenu des corrections de poids spécifiques de l'air les différences de pression peuvent être mesurées à \pm 2 à $3 / 10 \mathrm{~mm}$ près. 


\section{II. - MESURE DE VARIATIONS RAPIDES DE PRESSION}

Ce manomètre utilisé initialement pour la mesure de variations rapides de pression, consécutives à la fermeture d'une turbine, utilise la conpressibilité de l'air.

Il se compose essentiellement (fig, $n^{\circ} 6$ ) d'un tube de verre vertical fermé à son extrémité supérieure. L'extrémité inférieure est mise en communication avec l'eau sous pression qui refoule une certaine quantité d'air dans la partie supérieure du tube. met enfin de mesurer des fluctuations relativement rapides de pressions (jusqu'à des périodes de $1 / 5 \mathrm{~s}$ environ).

10 Sensibilité ET PRÉcision DU MANOMEtrRE.

L'équation reliant le déplacement du ménisque à la variation de pression peut s'écrire, d'après les notations de la figure $n^{\circ} 7$ :

$\mathrm{V}_{0}{ }^{\mathrm{K}} \mathrm{H}_{0}=\left[\mathrm{V}_{0}+s\left(x-x_{0}\right)\right]^{\mathrm{K}}\left(\mathrm{H}_{0}+d \mathrm{H}_{0}+x-x_{0}\right)$
FIG. 6.

SCHÉMA DE PRINCIPE DU MANOGMAPHE A Compression D'AIR.

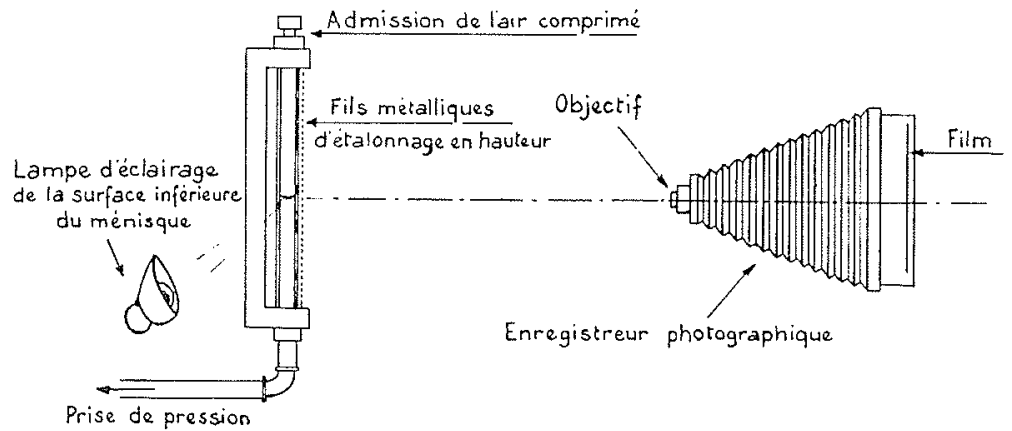

Suivant les fluctuations de la pression, l'air emprisonné dans le tube se comprime ou se détend; il en résulte une oscillation du ménisque de séparation de l'air et de l'eau, qui est enregistrée par dispositif photographique.

Outre sa grande simplicité de construction et d'empioi, cet appareil possède une excellente sensibilité, quelles que soient les pressions absolues et les variations relatives de pression à mesurer; sa faible constante de temps lui per-
IK étant un coefficient variant entre 1 et 1,4 suivant la nature des compressions ou détentes de l'air.

En négligeant la variation de pression de l'air due au déplacement du ménisque, cette relation peut se mettre sous la forme simplifiée:

$$
\left(1-\frac{s d x}{\mathrm{~V}_{0}}\right)^{\mathrm{K}}\left(1+\frac{d \mathrm{H}}{\mathrm{H}_{0}}\right)=1
$$

Frg. 7.

EQUation de DÉfinition DU MaNOGRAPHE a compression D'AIR.

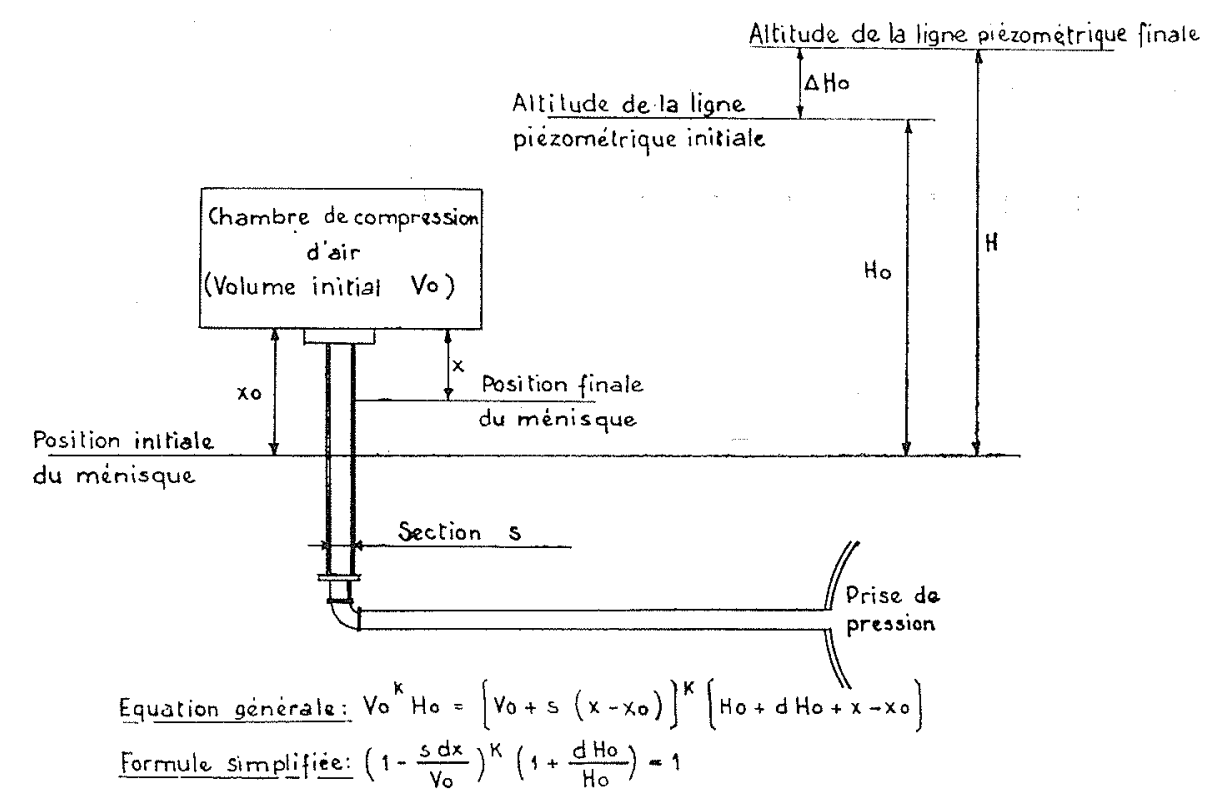

( $\mathrm{K}=$ coefficient dépendant de la nature des compressions et détentes de l'air $(1<\mathrm{K}<1,4)$ 
$d x$ représentant le déplacement du ménisque compté positivement vers le haut.

Cette expression montre que le déplacement du ménisque est indépendant de la pression absolue, mais dépend unicquement de la variation relative de pression, ce qui était évident a priori d'après la loi de Mariotte en régime isotherme. Elle montre, en outre, que lon peut toujours obtenir la pleine déviation de l'appareil pour une variation relative de pression quelconque donnée à l'avance : il suffit pour cela de choisir pour le volume $V_{0}$ une valeur calculée d'après l'équation ci-dessus.

La sensibilité de l'appareil est done excellente : il est possible de faire correspondre un déplacement de $5 \mathrm{~cm}$ du ménisque à une variation relative de pression de l'ordre de $1 \%$.

La fidélité du manomètre est elle-même excellente, sous réserve toutefois que la température de l'eau et celle de l'air ambiant restent constantes. Cela peut être réalisé aisément au cours de relevés de courté durée; pour les relevés s'étageant sur une durée de quelques heures, il y a lieu de prendre certaines précautions telles qu'une circulation d'eau à température constante autour du réservoir supérieur.

Le maintien d'une température constante exige en outre que les compressions ou détentes de l'air emprisonné dans le manomètre soient isothermiques.

Par suite de la faible constante de temps calorifique du manomètre (il suffit d'une seconde
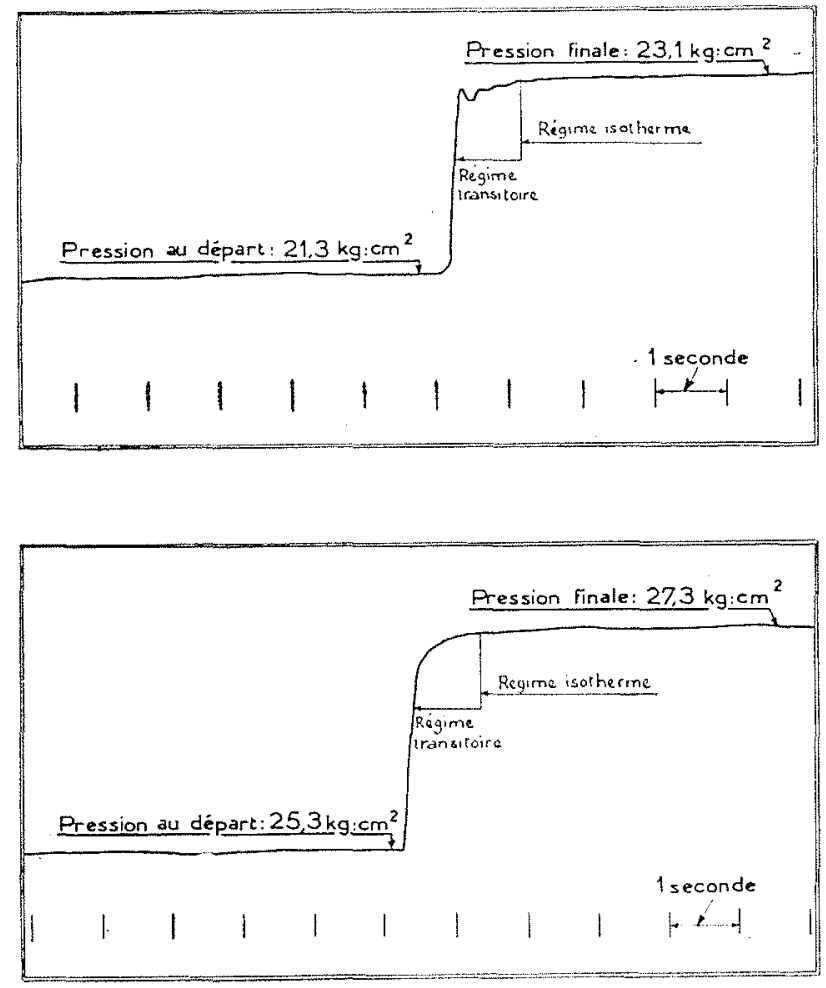

FIG. 8. pour que la chaleur accumulée dans la chambre de compression soit évacuée, ainsi qu'en témoignent les diagrammes de la fig. $n^{\circ} 8$ ), cela est pratiquement réalisé dans les mesures de la surpression créée dans une conduite forcée par la fermeture brusque du distributeur d'une turbine.

Pour la détermination de phénomènes plus rapides, il y a lieu d'apporter aux relevés bruts de l'appareil une correction variable avee la fréquence et l'amplitude des oscillations enregistrées, dont le calcul exact est malheureusement complexe. Une évaluation approchée de cette correction peut néanmoins être effectuée sans calcul; il en résulte une erreur de l'ordre de 5 à $10 \%$ de la variation relative de pression, ce qui correspond à une précision acceptable dans la plupart des cas (1).

Notons enfin que l'étalonnage ne dépend en fait que des dimensions géométriques de la chambre de compression.

Le tarage peut donc être effectué sous une pression absolue quelconque; il ne devra être renouvelé en principe que si l'on apporte une modification à la chambre de compression.

2. RÉPONSE DU MANOMĖTRE AUX VARIATIONS RAPIDES DE PRESSION.

On a vu précédemment que dans un manomètre soumis à une pression fluctuante, le coefficient reliant la grandeur à mesurer $\Delta h$ et la déviation de l'appareil de mesure $\Delta x$, était donné par la relation :

$$
\mathrm{K} \sim=\frac{\Delta h \sim}{\Delta x \sim}=Z_{\omega}
$$

En régime statique, réalisé au cours de l'étalonnage $(\omega=0)$, ce rapport devient :

$$
\mathrm{K}=\frac{\Delta h}{\Delta x}=\frac{1}{\mathrm{C}}
$$

L'erreur due à l'influence de l'inertie de l'eau et des frottements visqueux contre les parois des canalisations de raccordement, sera donc :

$$
\eta=\frac{\mathrm{K} \sim-\mathrm{K}=}{\mathrm{K}=}=\frac{Z_{\omega}-\frac{1}{\mathrm{C}}}{\frac{1}{\mathrm{C}}}=\left(\mathrm{C} Z_{\omega}-1\right)
$$

La réponse du manomètre sera donc d'autant plus satisfaisante que «l'impédance » et la

(1) En d'autres termes. la valeur instantanée d'une pression fluctuante, de pseudopériode inférieure à 1 seconde et d'une amplitude représentant $20 \%$ de la pression moyenne, pourra ctre déterminée avec une erreur. inférieure à \pm 1 à $2 \%$ près. 
« capacité » du manomèlre d'une part, la firiquence des oscillations de pressions d'autre part, seront faibles.

Or, les constantes d'inertic et de frottement $L$ et $R$ sont d'autant plus faibles, que les sections de canalisations de raccordement des prises de pression au manomètre sont grandes, par rapport à celles du tube dans lequel évolue le ménisque, En prenant pour ce dernier un diamètre de $2 \mathrm{~mm}$, l'influence de l'inertie et des frottements dans les canalisations de raccordement pourra être rendue négligeable dans la plupart des cas.

De ce fait, pour une hauteur maximum de $10 \mathrm{~cm}$ d'ean dans le tube de mesure, les constantes $L$ et $R$ auront respectivement pour valeurs :

$$
\begin{aligned}
& \mathrm{L}=0,01 \\
& \mathrm{R}=0,10
\end{aligned}
$$

La constante $G$ caractérisant les forces élastiques de rappel dépend seulement de l'amplitude de la variation de pression à mesurer. En effet, pour les faibles variations relatives de pression, on peut écrire en première approximation :

$$
\text { K.s. } \frac{d x}{\mathrm{~V}_{0}}=\frac{d \mathrm{H}}{\mathrm{H}_{0}}
$$

Si l'on introduit dans cette relation l'amplitude maximum $d \mathbf{H m}$ de la variation de pression mesurable avec le manometre et la dénivellation correspondante $d x m$ :

$$
\text { K. } s . \frac{d x m}{V_{0}}=\frac{d \mathrm{H} m}{\mathrm{H}_{0}}
$$

on en déduit :

$$
\frac{d x}{d x m}=\frac{d \mathrm{H}}{d \mathrm{Hm}}
$$

d'où :

$$
\frac{1}{\mathrm{C}}=\frac{d \mathrm{H} m}{d x m}
$$

La hauteur utile du tube de mesure est de $8 \mathrm{~cm}$, d'où :

$$
1=\frac{d \mathrm{Hm}}{0,08}
$$

$d \mathrm{H} m$ étant exprimé en mètres d'eau.

L'erreur $\eta$ due à l'inertie de l'eau et aux frot- tements visqueux contre les parois pourra s'écrire, après simplification :

$$
r_{i}=C \sqrt{\left(\frac{1}{C}-L_{\omega} \omega^{2}\right)^{2}+R^{2} \omega^{2}}-1
$$

Cette erreur restera inférieure à $1 \%$ lorsque la pulsation n'excédera pas à :

$$
\text { (1) }=\frac{1}{10 \sqrt{\mathrm{CL}-\frac{\mathrm{C}^{2} \mathrm{R}^{2}}{2}}} \leqslant 3 \sqrt{d \mathrm{Hm}}
$$

Pour une variation de pression, dont l'amplitude maximum est de $10 \mathrm{~m}$ d'eau, l'erreur due aux caractéristiques dynamiques du manometre n'excédera donc pas $1 \%$ si la pulsation $\omega$ est elle-même inférieure à 10 , ce qui correspond approximativement à une période de $1 / 2 \mathrm{sec}$ environ.

Aux variations de pression plus élevées, que l'on rencontre le plus souvent, cette limite de fréquence sera encore reculée.

\section{$3^{\circ}$ RÉalisation DU MANOMEtre.}

Le schéma de la figure $n^{*} 6$ donne les principes de la réalisation de cet appareil. On peut y voir notamment la chambre de compression d'air, munie de la valve d'admission ou d'échappement d'air comprimé, destinée au réglage initial de la position du ménisque sous la pression statique de référence. Le volume de cette chambre d'air peut être réduit, par introduction de cylindres métaliquues pleins, en vue de modifier la sensibilité du manomètre.

Le tube manométrique a un diamètre de $2 \mathrm{~mm}$ et une épaisseur de $4 \mathrm{~mm}$. Il peut résister à une pression de service de $200 \mathrm{~kg} / \mathrm{cm}^{2}$. L'erreur due à la capillarité de l'eau dans ce tube est négligeable devant la variation de pression que le manomètre doit normalement enregistrer.

Devant le tube de mesure est disposée unc grille conslituée de fils de cuive de $0,2 \mathrm{~mm}$ de diamètre, qui donnent sur le film photographique des droites constituant un étalonnage en hauteur, indépendant des dilatations de la pellicule.

Une lampe non ponctuelle éclaire une plage du ménisque, qui donne sur le film sensible une image plus on moins fine, suivant la vitesse et le sens du déplacement du méniscjue dans le tube (1).

Le dérouleur du film comporte une fente ver-

(1) Une étude pratique nous a permis de montrer que l'erreur due à la déformation du ménisque était négligeable; cela est dû au fajt que la plage lumineuse du ménisque se situe au voisinage du centre de gravité de ce dernier. Pour les variations relativement lentes de pression, la déformation du ménisque est d'ailleurs très peu importante en raison du faible diamètre du tube. 
licale mince, limitant l'épaisseur instantanée de l'image dans le sens du dérouleur du film. Il est muni, en outre, d'un dispositif de marquage de temps constitué par un éclateur, alimenté par le secondaire d'un transformateur élévateur, dont le primaire est périodiquement interrompu par un batteur de secondes mécanique.

Les enregistrements obtenus sont satisfaisants, malgré la grande simplicité de cet appareil.

\section{CONCLUSION}

Le manomètre différentiel à colonnes « aireau $\gg$ et le manographe à compression d'air, complétant le matériel courant de la Division des Essais Extérieurs, nous ont permis de résoudre, avec une précision suffisante et au prix de frais relativement modestes, la plupart des problèmes usuels rencontrés au cours des essais d'installations hydroélectriques. Il y aurait peutêtre lieu d'ajouter à ces deux appareils, un manomètre différentiel à transmission par air, actuellement en cours de réalisation et qui serait destiné notamment au relevé d'altitude de la ligne piézométrique dans les cheminées d'équilibre, dont l'ouverture supérieure constitue le seul accès rapproché.

Parmi ces appareils, le manographe à compression d'air a perdu évidemment beaucoup de son intérêt depuis l'apparition, dans la technique de mesure, des appareils électroniques, aux qualités nettement supérieures, tant par leur sensibilité et leur faible constante de temps, que par la linéarité de leur loi de réponse. Tontefois, la simplicité de mise en œuvre et de construction du manographe à compression d'air, lui permettra peut-être de rendre encore quelques services dans l'avenir.

\section{I S C U S S I O N}

\section{1" MANOMETRE A COLONNE AIR-EAU :}

M. Ie Président souligne l'intérêt de cet appareil et rappelle qu'il est une des applications des considérations theoriques exposées par M. Valembors dans sa thèse : Mesure des pressions bariables.

M. Dupouy demande s'il n'y aurait pas intérêt à remplacer l'air par un liquide plus léger que l'eau et qui serait incompressible (huile, par exemple), ce qui atténuerait les fluctuations et les effets de résonance, et augmenterat la sensibilite (manometre à deux liquides).

i. Bounaugax répond que le manometre à deux liquides envisage par M. Dupour convient aux Laboratoires. mais qu'il serait peu fidéle dans les applications industrielles où la turbidité des eaux, la variation brusque de pression et les fuites de canalisations soulèvent de nombreuses difficultés.

\section{2" MANOMETRE FNREGISTREUR A COMPRESSION D'AIR :}

M. le Président remereie M. Bovnguavon de son intéressante communication.

M. Texot demande si ce manomètre enregistreur est applicable au cas de période extremement courtes bien inférieures à $1 / 6$ de seconde comme celles intervenant dans les coups de bélier d'ondes relatifs aux pompes centrifuges.

M. Boungurgnon croit qu'il en est bien ainsi, et ajoute qu'à sa comnaissance, seul le manomètre piézoélectrique $\dot{a}$ quartz est effectivement susceptible de mesurer exactement des fluctuations très rapides.

M. Reneneras souligne que les appareils décrits par
M. Bourguigion ont été construits pour des essais industriels, notamment le manomètre différentiel à colonnes air-eau utilisé pour le contrôle permanent des débits dans les conduites par la mesure de la différence de pression entre les deux extrémités $A$ et $B$ d'un conversent.

En pratique les pressions en $A$ et en $B$ ne sont pas constantes dans le temps mais présentent des flucturtions incessantes dont la période est de quelques secondes. Schématiquement la pression $P$, pourait être représentée par une expression de la forme:

$$
\mathrm{P}_{\mathrm{A}}=\mathrm{P}+p \sin \omega t
$$

L'amplitude maximum $p$ de la composante « alternative» de la pression peut être de quesques « pour cent» de celle de la composante « continue »P. Dans ces conditions la mesure précise avec un manomètre à colonne d'eau d'une faible valeur de $P_{A}-P_{P}$, pour de fortes valeurs de $p$ est très difficile, les deux ménisques pouvant osciller sensiblement en synchronisme avec unc amplitude du même ordre de grandeur que la dénivellation à mesurer.

Le manomètre differentiel réalisé par M. Bouraulinow permet de ramener a une valeur trés faible la valeur do la composante alternative $p$ sin $\omega t$ par un systeme d'amortissement spécialement étudié pour ne pas introduire d'ereur; on se trouve alors ramené ì la mesure de la différence de deux pressions constantes dans le temps. Le dispositif employé peut trouver des applications dans divers systèmes de régulation ou de commandes automatiques.

M. Ie Président remercie M. Remenieras de ce complément à la communication de M. Boungugson. 